\title{
Development of a computational tool for materials design
}

\author{
Shuang-Lin Chen • Wei-Sheng Cao • \\ Fan Zhang $\cdot$ Chuan Zhang $\cdot$ Jun Zhu \\ Jie-Yu Zhang
}

Received: 15 January 2013/Accepted: 23 April 2013/Published online: 23 May 2013

(C) Shanghai University and Springer-Verlag Berlin Heidelberg 2013

\begin{abstract}
An integrated modeling tool coupling thermodynamic calculation and kinetic simulation of multicomponent alloys is developed under the framework of integrated computational materials engineering. On the basis of Pandat ${ }^{T M}$ software for multicomponent phase diagram calculation, the new tool is designed in an integrated workspace and is targeted to understand the composition-processing-structure-property relationships of multicomponent systems. In particular, the phase diagram calculation module is used to understand the phase stability under the given conditions. The calculated phase equilibrium information, such as phase composition and chemical driving force, provides input for the kinetic simulation. In this paper, the design of the modeling tool will be presented and the calculation examples from the different modules will also be demonstrated.
\end{abstract}

Keywords Phase diagram - Materials design · Ni alloy · Ti alloy $\cdot$ Diffusion simulation $\cdot$ Precipitation simulation

\section{Introduction}

Optimization of alloy chemistry and processing conditions is a common practice of materials scientists/engineers in the design of new materials and the improvement of the existing ones. Traditionally, this process was accomplished

S.-L. Chen $(\bowtie) \cdot$ W.-S. Cao · F. Zhang · C. Zhang · J. Zhu CompuTherm LLC, 437 S. Yellowstone Dr., Madison, WI, USA e-mail: shuanglin.chen@ hotmail.com

S.-L. Chen · J.-Y. Zhang

School of Materials Science and Engineering, Shanghai University, Shanghai 200072, People's Republic of China through a trial-and-error approach, which is costly and time-consuming. With the recent advancement of the computational materials engineering and modern information technology, alloy design process can be greatly accelerated with the aid of modeling tools. Different types of modeling tools have been developed in recent years which range from the atomic level to the continuum level and from the thermodynamic models to the physics-based property models. Each individual model is used to understand a particular procedure or phenomenon. The vision for integrated computational materials engineering (ICME) is to develop a comprehensive, integrated suite of validated computational materials models linked to analysis systems for manufacturing processes and engineering design [1]. If such a suite is developed, it can unlock the great potential for significant benefits in cost-effective materials and process design. However, in order to develop the integrated suite, each individual model should be thoroughly validated first.

Phase diagram calculation based on the CALPHAD approach [2-4], which is used to understand the effect of the alloy chemistry on the final microstructure, is certainly one of the key stones of the ICME suite. The essence of the CALPHAD approach is to obtain self-consistent thermodynamic descriptions of the lower order systems: binaries and ternaries, in terms of the known thermodynamic and phase equilibrium data. The self-consistence means that the separate measured and calculated thermodynamic data, as well as the phase equilibrium data, can be described by the same thermodynamic description. Moreover, the thermodynamic description, or called thermodynamic database, of higher order systems can be developed using those of the lower order systems and geometric models. This database enables us to calculate the phase equilibrium of the multicomponent systems those are experimentally unavailable. 
Even though the CALPHAD approach is a phenomenological approach, it has become a widely accepted tool to ICME practitioners. This is because it is by far the only approach that allows the calculation of the phase equilibrium in the technically important multicomponent alloys.

While the phase diagram calculation can predict the stable phases and the amount of each phase in the equilibrium microstructure, it cannot simulate the evolution of the microstructure as a function of time and processes. Kinetic simulation is needed to understand the detailed microstructure features given the alloy chemistry and processing conditions. The CALPHAD approach has, therefore, been extended to a broader field of materials science and engineering beyond the phase diagrams, such as diffusion simulation and precipitation simulation. Mobility database has been developed in a similar manner as that of thermodynamic database. The integrated thermodynamic and mobility databases can then be used to simulate the diffusion-controlled kinetic processes. In the precipitation simulation, for example, the phase equilibrium information and the chemical driving force are obtained from the phase diagram calculation, which are then used as the input of the kinetic models those account for the simultaneous nucleation, growth and coarsening for the simulation of microstructural evolution. The simulated microstructural features include the volume fraction, the average particle size, the number density, and the particle size distribution. Such data can further be used as the input for the mechanical property models and enable one to calculate the alloy's mechanical properties.

In this paper, we will present Pandat ${ }^{T M}$ software package, a modeling tool developed on the basis of the CALPHAD approach. The design of this modeling tool follows the concept of the ICME, which integrates the thermodynamic calculation with kinetic simulation in a seamless manner. This tool can be used to perform the calculation of the phase equilibrium, diffusion, and the precipitation of the multicomponent systems. The simulation results provide the key information for understanding the effects of the alloy chemistry and processing conditions on the developed microstructure and materials properties. The paper will be arranged as follows: the software design will be presented in the first part, the calculation examples from the different modules will be demonstrated in the second part, and the conclusions will be summarized in the last part.

\section{Software design}

In this section, we will present the design of the Pandat ${ }^{T M}$ software, its architecture, its variety of modules, and its calculation engine.

\subsection{Architecture}

Pandat $^{T M}$ software is designed as a workspace, which may include multiple modules for performing the variety of the simulations and be easily extended. Currently, four modules are available, which are PanPhaseDiagram for multicomponent phase diagram calculation, PanOptimizer for thermodynamic model parameter optimization, PanPrecipitation for precipitation simulation, and PanDiffusion for diffusion simulation. The architecture of Pandat ${ }^{T M}$ software is shown in Fig. 1, which consists of three layers: the interface layer, the calculation layer and the database layer. The top layer, i.e., the interface layer, is responsible for the communication between the user and the software. It passes the user commands and calculation conditions to the calculation layer and displays the calculated results returned from the calculation layer in the format of table or graph. The middle layer, i.e., the calculation layer, processes the user commands and calculation conditions from the interface layer, load the needed model parameters from the database layer, perform the calculation, and then return the calculated results back to the interface layer. The bottom layer, i.e., the database layer, stores all the model parameters needed by the calculation.

\subsection{PanEngine}

It should be mentioned that the calculation engine, PanEngine, is shared by the different modules in the calculation layer as shown in Fig. 1. PanEngine, which perfoms all the thermodynamic calculations required by different modules, is a dynamic linked library. PanEngine can therefore be integrated with other models through its application programming interface (API) and create many

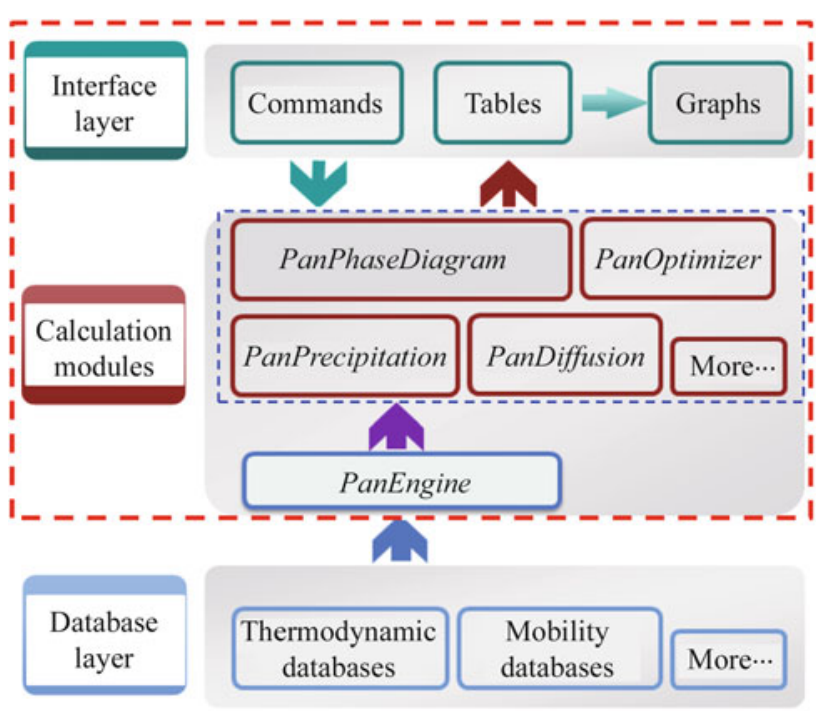

Fig. 1 Architecture of Pandat ${ }^{T M}$ software 


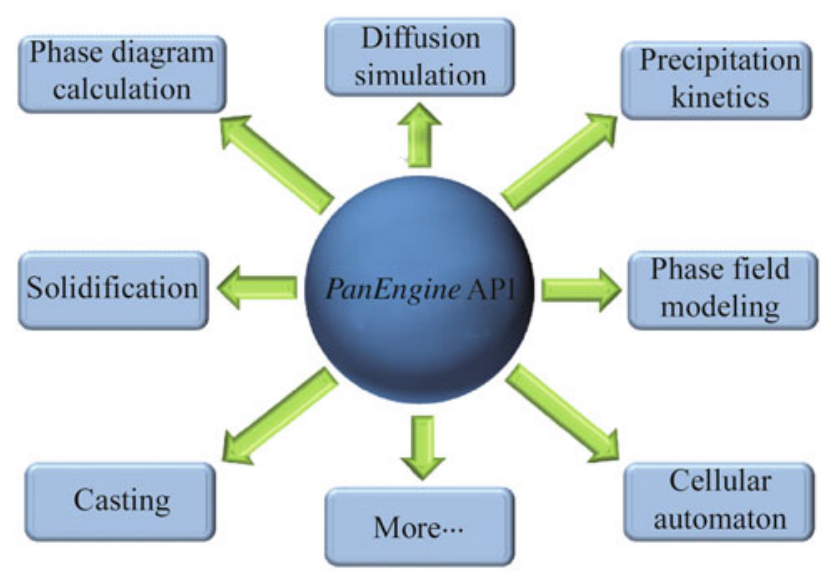

Fig. 2 Applications through the integration with PanEngine API

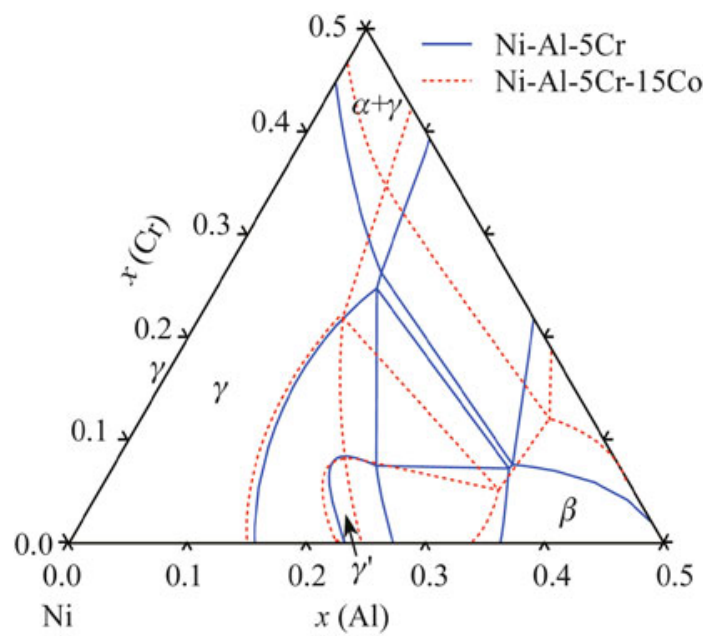

Fig. 3 Isotherm of $\mathrm{Ni}-\mathrm{Al}-5$ at. $\% \mathrm{Cr}$ at $1,000{ }^{\circ} \mathrm{C}$ with 15 at.\% $\mathrm{Co}$ and without $\mathrm{Co}$

customized applications, as shown in Fig. 2. This is a good platform for the development of the ICME tools.

\section{Application examples}

In this section, we will use examples to demonstrate the applications of different modules of Pandat ${ }^{T M}$ software in predicting the multicomponent phase equilibrium, the diffusion and the microstructural evolution.

\subsection{Phase diagram calculation module}

Phase diagram calculation module, PanPhaseDiagram, is used to calculate the multicomponent phase equilibria and related thermodynamic properties. The calculation can be performed for a point with the fixed composition and temperature, for a series of points along a line with varying

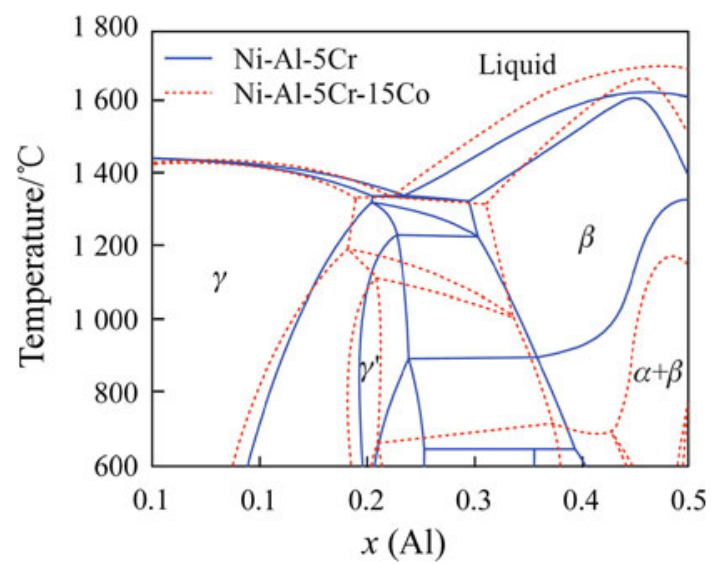

Fig. 4 Isopleth of Ni-Al-5 at.\%Cr between $600{ }^{\circ} \mathrm{C}$ and $1,000{ }^{\circ} \mathrm{C}$ with 15 at.\% Co and without Co

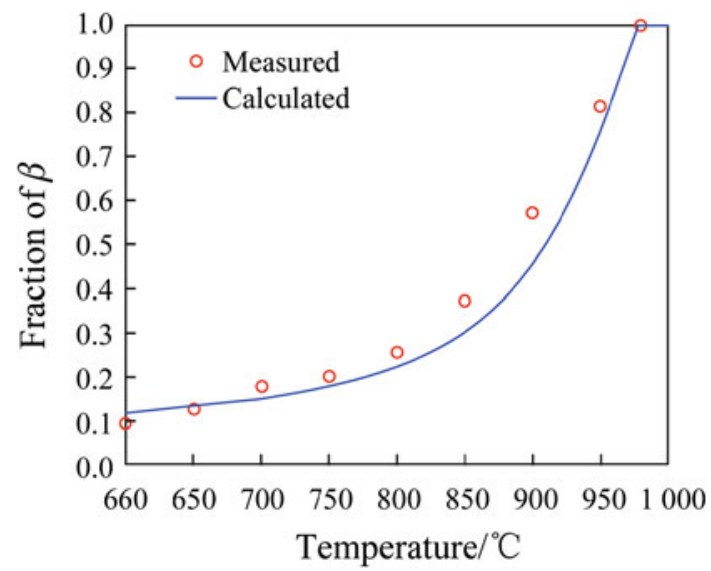

Fig. 5 Calculated $\beta$-approach curve for Ti64 alloy with the composition of Ti-5.72Al-4.04V-0.147Fe-0.08O-0.034C-0.016N-0.0063H (wt\%) and the experimental data are from Castro and Seraphin [5]

temperature or composition, or for a two dimensional diagram. The calculated properties can be phase equilibrium, liquidus projection, solidification, and variety of the thermodynamic properties, such as heat of evolution and activities. In the following, we will demonstrate how we can use these calculations to understand the composition effect on materials properties.

\subsubsection{Nickel alloy}

As is known, $\mathrm{Al}$ and $\mathrm{Cr}$ are the two basic alloying elements of nickel-based superalloys, and $\mathrm{Ni}-\mathrm{Al}-\mathrm{Cr}$ is the major ternary system in the design of the nickel alloys. In recent years, high percentage of Co is used in the latest generation of nickelbased superalloys. Even though our primary focus of this paper is not to understand the effect of Co on the properties of nickel-based superalloys, we will use the phase diagram calculation to see its effect on the phase stability of the 


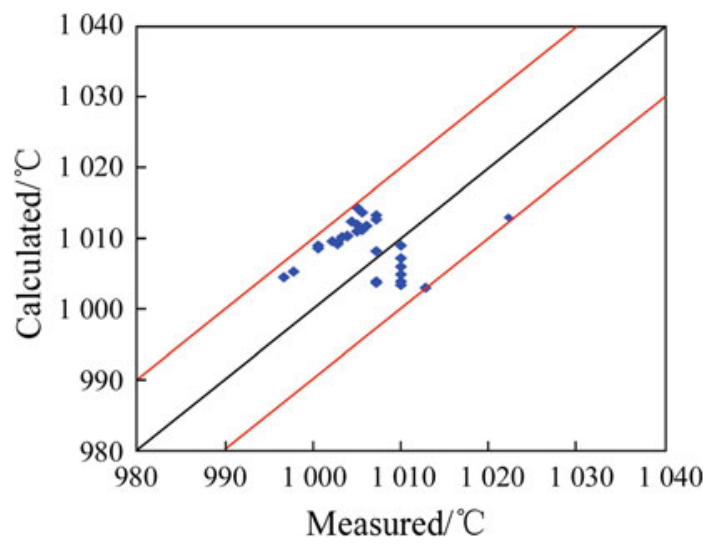

Fig. 6 Comparison of calculated and measured $\beta$-transus for a group of 30 Ti6242 alloys

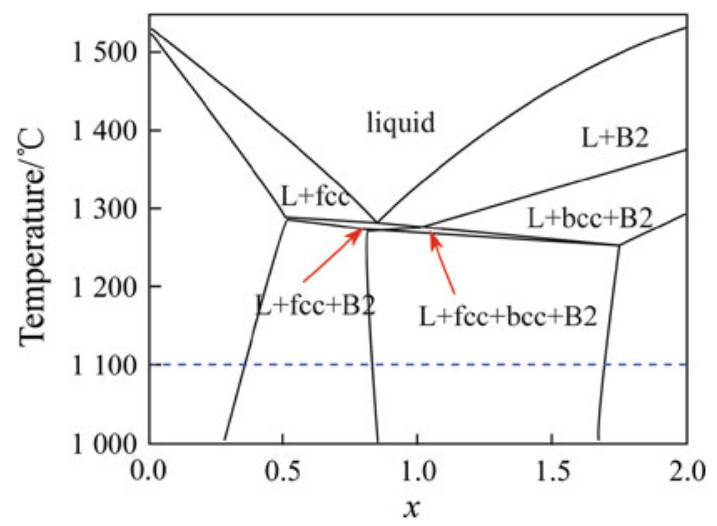

Fig. 7 Calculated isopleth of the $\mathrm{Al}_{x} \mathrm{CoCrFeNi}$ alloys with $x$ in the range of $0-2$

$\mathrm{Ni}-\mathrm{Al}-\mathrm{Cr}$ system. Figure 3 shows the effect of Co on the isotherm of Ni-Al-Cr at $1,000{ }^{\circ} \mathrm{C}$. The red dash line outlines the phase boundary when 15 at.\% of Co is used. It is seen that Co increases the stable field of the single $\beta$ phase, while decreases that of the $\gamma^{\prime}$ phase. Figure 4 compares the isopleths of Ni-Al-5 at.\%Cr with 15 at.\% of Co and without Co. The same conclusion, Co increases the stability of the $\beta$ phase and decreases that of the $\gamma^{\prime}$ phase, is obtained. Since the phase stability plays a key role in the determination of the microstructure, and eventually the properties of a material, multicomponent phase diagram calculation therefore provides the insight on understanding the effects of the newly added elements and guides materials design.

\subsubsection{Titanium alloy}

Technical multicomponent titanium alloys are classified as alpha $(\alpha)$, beta $(\beta)$, and alpha-beta $(\alpha+\beta)$ alloys depending on the relative amount of $\alpha$ - and $\beta$ - stabilizing components in the alloy. The microstructure and mechanical

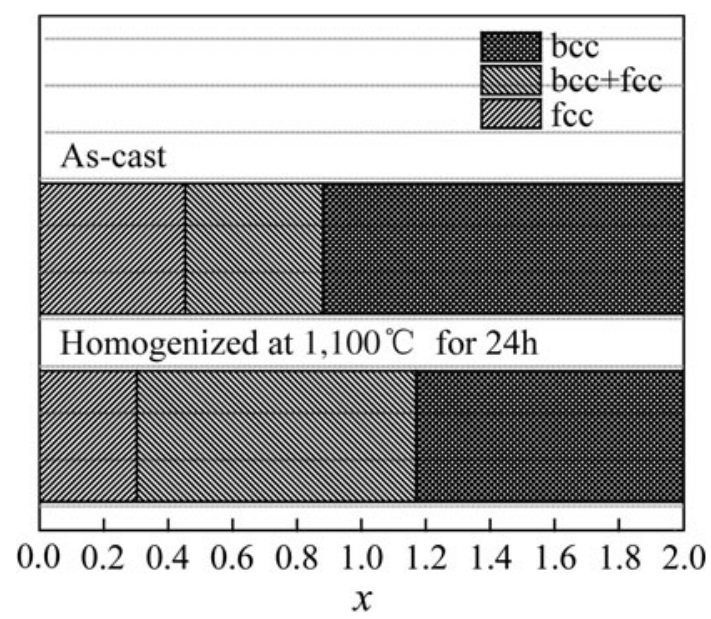

Fig. 8 Experimentally measured transition ranges of the $\mathrm{Al}_{x} \mathrm{CoCrFeNi}$ alloys by Kao et al. [6]

properties of a titanium alloy strongly depend on the alloy chemistry. To understand their correlations, multicomponent phase equilibrium information is needed. Two important pieces of information needed for selecting heat treatment condition are the $\beta$-approach curve and $\beta$-transus temperature. $\beta$-approach curve is the curve showing the fraction of $\beta$ phase as a function of temperature, and the $\beta$-transus is the temperature at which $\alpha$ phase starts to precipitate from the $\beta$ phase.

Figure 5 shows the calculated $\beta$-approach curve for one Ti64 alloy with experimentally data plotted for comparison. It should be pointed out that, the nominal composition of Ti64 is Ti-6Al-4V (wt\%), however, the composition of the commercial Ti64 alloy varies in a composition range. Moreover, minor alloying elements, such as $\mathrm{Fe}, \mathrm{O}, \mathrm{C}, \mathrm{N}$, and $\mathrm{H}$, are usually included in the alloy. It is found that the $\beta$-approach curve strongly depends on the alloy chemistry, the capability of being able to calculate such curve given alloy chemistry is therefore important in the selection of the heat treatment parameters. The alloy composition used to calculate the $\beta$-approach curve of Fig. 5 is Ti-5.72Al$4.04 \mathrm{~V}-0.147 \mathrm{Fe}-0.08 \mathrm{O}-0.034 \mathrm{C}-0.016 \mathrm{~N}-0.0063 \mathrm{H}$ (wt $\%$ ). The experimental data are from Castro and Seraphin [5].

Figure 6 shows a comparison between the calculated and measured $\beta$-transus temperature for a group of Ti6242 alloys. Again, the nominal chemistry of Ti6242 alloy is Ti6Al-2Sn-4Zr-2Mo (wt\%), while the chemistry of commercial Ti6242 may vary and include other minor elements, such as $\mathrm{Fe}, \mathrm{Si}, \mathrm{C}, \mathrm{N}, \mathrm{O}$, and $\mathrm{H}$. The $\beta$-transus temperature may vary between $990{ }^{\circ} \mathrm{C}$ and $1,020{ }^{\circ} \mathrm{C}$ depending on the alloy composition. As shown in Fig. 6, the calculated $\beta$-transus temperature agrees with the experimental measured one fairly well for a group of 30 Ti6242 alloys. 


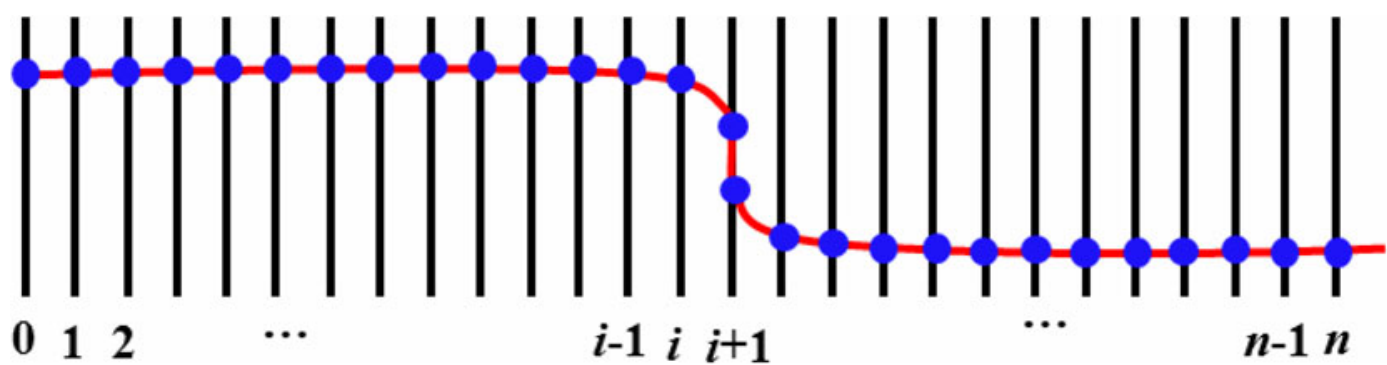

Fig. 9 Schematic diagram showing the concentration profile of a component in a diffusion couple

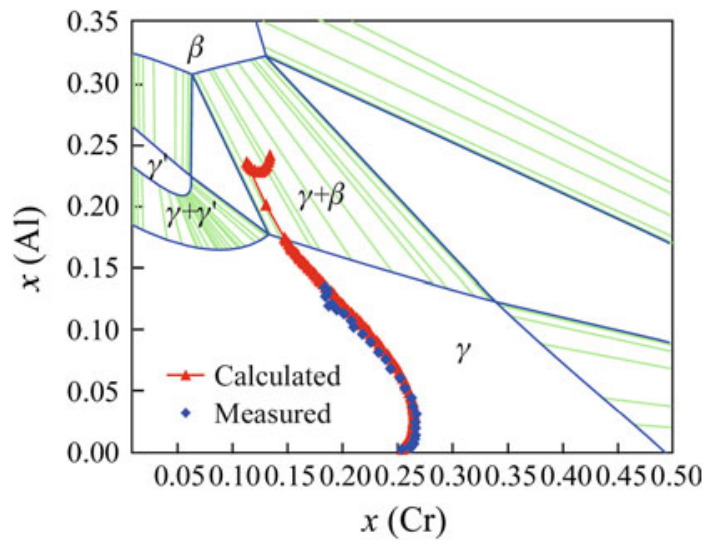

Fig. 10 Comparison of the calculated diffusion path with experimental data on the isotherm of Ni-Al-Cr ternary system at $1,200{ }^{\circ} \mathrm{C}$

\subsubsection{High entropy alloy}

High entropy alloy (HEA), representing a new concept of the alloy design, has revolutionized the traditional alloy design approach that is usually based on one or, at most two key elements. On the contrary, HEAs have multiprincipal elements which are in equal or near to equal atomic ratios. It is claimed that the HEAs tend to form the simplified solid solution structure, such as fcc or bcc, or a mixture of both. This tendency is due to the high entropy of mixing of the solution phases. As is known, the stability of a phase is determined by its Gibbs energy which includes contributions from both enthalpy and entropy. The stable phases in a system under a given condition are the result of the stability competition among all the phases. Phase diagram calculation, therefore, is a powerful tool in the design of HEAs since it can calculate multicomponent phase equilibria. Figure 7 shows the phase stability change when adding $\mathrm{Al}$ to the $\mathrm{CoCrFeNi}$ alloy which compares favorably with the experimental observation by Kao et al. [6] (see Fig. 8). Both indicate that $\mathrm{Al}$ promotes the formation of bcc structure. The calculation shows a wider composition range which possesses the fcc + bcc two-phase mixture than that shown by the experiment. However, the experiment did show an enlarged fcc + bcc two-phase composition range for the homogenized alloy comparing to

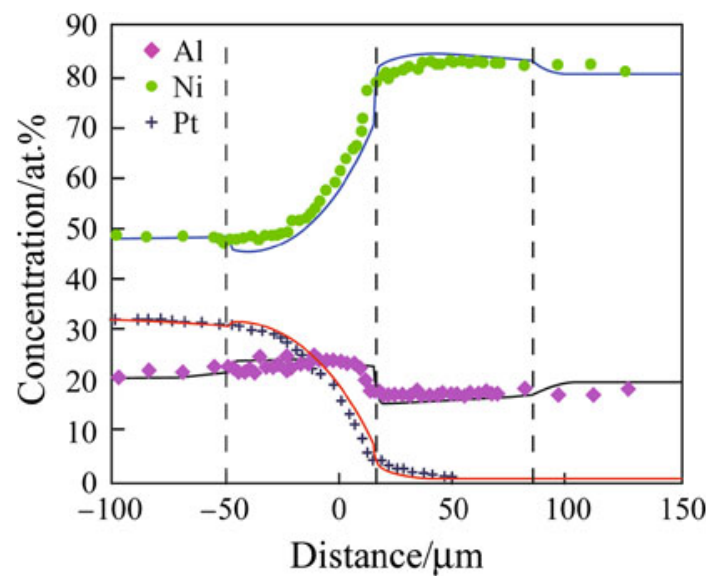

Fig. 11 Comparison of simulated concentration profiles with experimental data [8] for the Ni-22Al-30Pt/Ni-19Al (at.\%) diffusion couple after $50 \mathrm{~h}$ of interdiffusion at $1,150{ }^{\circ} \mathrm{C}$

the casting alloy. Should the alloy be annealed longer time, the wider fcc + bcc two-phase composition range might be observed.

\subsection{Diffusion simulation module}

Diffusion simulation module, PanDiffusion, has been developed in Pandat ${ }^{T M}$ software for the diffusion simulation. This module was developed based on 1D diffusion model. As shown in Fig. 9 schematically, the diffusion couple is divided into small slice as represented by grids. The diffusion is initiated due to the chemical potential difference of a component on the neighbor grids. At each time step, the diffusion flux is calculated and the composition of each grid point updated. The stable phase equilibrium at each grid will then be calculated using the updated composition. The phase equilibrium calculation is directly coupled with the thermodynamic calculation engine, PanEngine, and the update is instant at each time step.

Diffusion simulation is very useful in understanding the diffusion controlled processes and the resulting microstructure. One application is for the development of advanced coating of metal alloys, in which the diffusion between the coating and the substrate needs to be fully 


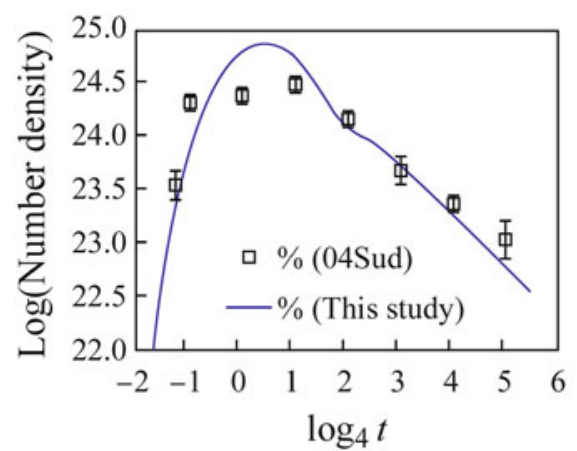

(a)

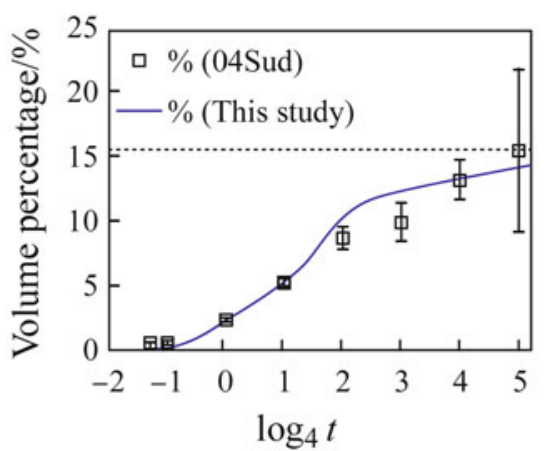

(b)

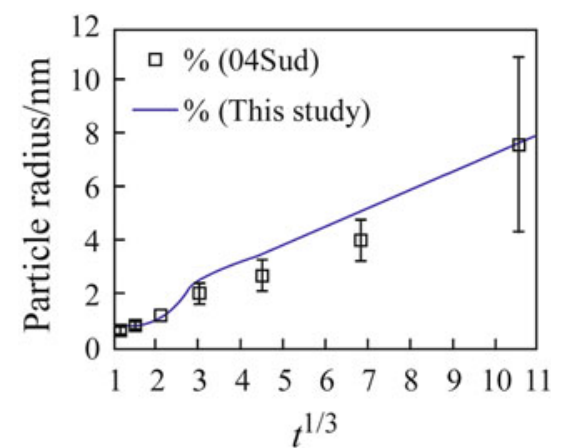

(c)

Fig. 12 Comparison of predicated temporal evolution of $\gamma^{\prime}$ a number density in log scale, $\mathbf{b}$ volume fraction, $\mathbf{c}$ mean size versus $t^{1 / 3}$ for alloy Ni-5.2 $\mathrm{Al}-14.2 \mathrm{Cr}$ (at.\%) aged at $600{ }^{\circ} \mathrm{C}$ with the experimental data [9]

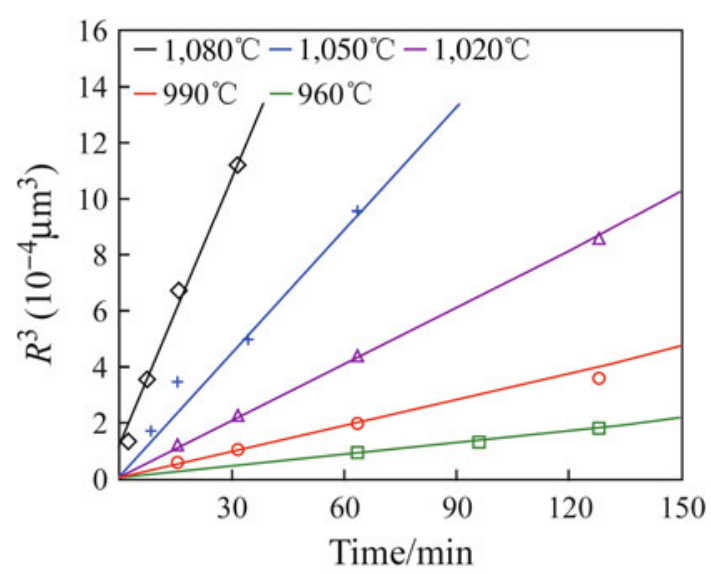

Fig. 13 Predicated temporal evolution of mean $\gamma^{\prime}$ size in alloy Rene88DT aged at $960{ }^{\circ} \mathrm{C} ; 990{ }^{\circ} \mathrm{C} ; 1,020{ }^{\circ} \mathrm{C} ; 1,050{ }^{\circ} \mathrm{C}$ and $1,080{ }^{\circ} \mathrm{C}$ compared with the experimental data [10]

understood. The diffusion module can be used to calculate the diffusion path given two composition points. Figure 10 shows such an example in the Ni-Al-Cr ternary system. The red line is the simulated diffusion path, while the blue points are experimental determined data [7].

The PanDiffusion module can also be used to calculate the concentration profiles of a diffusion couple. Figure 11 compares the calculated concentration profile with the measured data for the Ni-22Al-30Pt/Ni-19Al (at.\%) diffusion couple after $50 \mathrm{~h}$ interdiffusion at $1,150{ }^{\circ} \mathrm{C}$. As is shown, the calculated concentration profiles agree with the experimental data very well [8].

\subsection{Precipitation simulation module}

Precipitation hardening provides one of the most widely used mechanisms for the strengthening of many structural materials. Modeling of precipitation process, therefore, plays an important role in understanding the behavior of materials. However, precipitation is a complex process involving the simultaneous occurrence of nucleation, growth and coarsening. Accurate modeling of the precipitation process requires a synchronous consideration of all these contributions to simulate the temporal evolution of microstructure and the corresponding responses of the mechanical properties. Moreover, the phase equilibrium information as well as the composition and mobility data of the matrix phase need to be constantly updated as the nucleation, growth and coarsening proceed. Such a simulation, therefore, requires a smooth integration of the thermodynamic calculation, kinetic simulation and property modeling of the material. Pandat ${ }^{T M}$ software provides such an integrated workspace, and PanPrecipitation module is directly integrated with the thermodynamic calculation engine, PanEngine.

Figure 12 shows an example of precipitation simulation for alloy Ni-5.2Al-14.2Cr (at.\%) aged at $600{ }^{\circ} \mathrm{C}$. Figure $12 \mathrm{a}$ shows the simulated temporal evolution of $\gamma^{\prime}$ number density compared with the measurements [9]. The number density increases in the early stages, reaches a maximum of $7 \times 10^{24}$ and subsequently decreases due to coarsening. Figure $12 \mathrm{~b}$ shows the temporal evolution of $\gamma^{\prime}$ volume fraction, which gradually approaches the equilibrium fraction of $15.8 \%$. Figure $12 \mathrm{c}$ indicates the mean radius initially remains essentially the same, and begins to grow after $1 \mathrm{~h}$ before coarsening starts. Eventually, the coarsening effect becomes dominant after $16 \mathrm{~h}$. After a long aging time of $1,024 \mathrm{~h}$, the mean radius is around $7.7 \mathrm{~nm}$ and the equilibrium volume fraction $15.8 \%$ is nearly attained.

Another example is shown for the simulation of the coarsening behavior in a commercial alloy Rene88DT (Ni-1.99Al-12.99Co-15.67Cr-4.00Mo-3.88W-3.72Ti-0.70Nb in wt \%). Figure 13 shows the cube of the predicated mean $\gamma^{\prime}$ size against time for five different temperatures $1,080{ }^{\circ} \mathrm{C}$; $1,050{ }^{\circ} \mathrm{C} ; 1,020{ }^{\circ} \mathrm{C} ; 990{ }^{\circ} \mathrm{C}$ and $960{ }^{\circ} \mathrm{C}$, which show good agreement with the measured values by Mao [10], the 
coarsening rate rises as the temperature increases. For each temperature, it is found that the $\gamma^{\prime}$ coarsening kinetics is consistent with the LSW theory even when the holding time is short, i.e., $R^{3}$ is proportional to time.

\section{Conclusions}

The lack of the effective computational tools for materials engineering has become an obstacle for accelerated materials design and development. The core of ICME is to develop the integrated models and databases which can quantitatively describe the chemistry-processing-structure-property relationships and be used by the engineering community to solve the materials development and application problems.

In this work, we have demonstrated our efforts in the development of Pandat ${ }^{T M}$, a computational tool based on the CALPHAD approach. This modeling tool, which smoothly integrates the computational thermodynamics and kinetics in one workspace, allows user to develop a big picture in understanding the effects of alloy chemistry and processing conditions on the final microstructure and properties of a material. This modeling tool is designed for the multicomponent systems and can be directly applied to the commercial alloys, such as nickel-based superalloys and titanium alloys. Moreover, the capability of this modeling tool can be easily extended due to its generic data structure.

\section{References}

1. Allison J, Backman D, Christodoulou L (2006) Integrated computational materials engineering: a new paradigm for the global materials profession. J Manag 11:25-27

2. Kaufman L, Bernstein H (1970) Computer calculation of phase diagrams. Academic Press, New York

3. Saunders N, Miodownik AP (1998) CALPHAD: a comprehensive guide. In: Cahn RW (ed) Pergamon materials series: V1. Elsevier, Oxford

4. Chang YA, Chen SL, Zhang F et al (2004) Phase diagram calculation: past, present and future. Prog Mater Sci 49:313-345

5. Castro R, Seraphin L (1966) Mémoiresscientifiques de la revue de métallurgie. Mem Sci Rev Met 63:1025-1058

6. Kao YF, Chen SK, Chen TJ et al (2011) Electrical, magnetic, and hall properties of $\mathrm{Al}_{x} \mathrm{CoCrFeNi}$ high-entropy alloys. J Alloys Compd 509(5):1607-1614

7. Nesbitt JA, Heckel RW (1987) Interdiffusion in Ni-Rich, Ni-Cr-Al alloys at $1000{ }^{\circ} \mathrm{C}$ and $1200{ }^{\circ} \mathrm{C}$ : part I. Diffusion paths and microstructures. Metall Trans A 18(12):2061-2073

8. Gleeson B, Wang W, Hayashi S et al (2004) Effects of platinum on the interdiffusion and oxidation behavior of Ni-Al-based alloys. Mater Sci Forum 461-464:213-222

9. Sudbrack CK (2004) Decomposition behavior in model Ni-AlCr-X superalloys: temporal evolution and compositional pathways on a nanoscale. Dissertation, Northwestern University, Chicago

10. Mao J (2002) Gamma prime precipitation modeling and strength responses in powder metallurgy superalloys. Dissertation, West Virginia University, Morgantown 